\title{
Algebra-Based Students and Vector Representations: Arrow vs. ijk
}

\author{
John B. Buncher \\ Department of Physics, North Dakota State University, Fargo, ND, 58108, USA
}

\begin{abstract}
A recent study in a calculus-based introductory physics course found that students performed significantly better on vector addition and subtraction tasks when questions were given in the $i j k$ representation instead of an "arrows-on-a-grid" representation. This study also presented evidence that working knowledge of the $i j k$ format was necessary to correctly perform vector operations in the arrow format, which has instructional implications for algebra-based introductory physics, as many associated courses and texts extensively use the arrow format and neglect the $i j k$ format. We conducted a study of students in an algebra-based introductory physics course where students were given no explicit instruction on the $i j k$ format, but had received instruction on the arrow format. We find that at the end of the semester algebra-based introductory physics students perform significantly higher in the $i j k$ representation than in the arrow representation on both one- and two-dimensional problems. Our findings imply that these students may also benefit from the use of $i j k$ in class or as part of instruction.
\end{abstract}

PACS numbers: $01.40 . \mathrm{Fk}, 01.40 . \mathrm{gb}$

Keywords: vectors, arrow representation, algebra-based, introductory physics

\section{INTRODUCTION}

Vector addition and subtraction is an essential component to correctly analyzing many topics covered in a typical introductory physics sequence. Student difficulties with vector operations have been extensively documented [1-8], and this work aims to add to our understanding of their difficulties and identify possible underutilized student resources in the context of an algebra-based introductory physics course at a large public research university.

Students typically enter a physics course with little useful vector knowledge $[1,2]$. While instruction can lead to gains in performance, vector addition and subtraction tasks remain quite difficult for students in both algebra- and calculusbased courses after one semester of instruction, and this deficiency leads to further difficulties in mastering the physics content [2-4]. Further investigations into these difficulties have shown: the relative position and context of vectors can cue a particular solution (or error) [5, 6], students tend to stick with their initial solution method [7], and that student performance on one-dimensional vector subtraction is affected by the vector's relative orientation [8]. Each of these studies examined student difficulties with vectors primarily in the arrow representation, which leaves other vector representations such as $i j k$ underrepresented in the literature.

The lack of studies on student performance in the $i j k$ representation, and specifically a comparison of performance between the $i j k$ and arrow representations, was noted by Heckler et al. in a paper aimed at addressing this particular issue [9]. Heckler found that students in a calculus-based introductory course performed significantly better on vector addition and subtraction in the $i j k$ representation compared to the arrow representation, and found evidence of a knowledge hierarchy: success on vector operations in the $i j k$ representation was necessary for success in the arrow representation.

The presence of this knowledge hierarchy presents an interesting question: would students in an algebra-based course benefit from instruction in the $i j k$ format? Heckler notes that while "it is possible that the $i j k$ format may prove to be too formal and unfamiliar for ... less mathematically prepared stu- dents", their findings suggest that the $i j k$ representation "is more naturally and hierarchically fundamental for applying vector sum and difference operations". A sampling of popular algebra-based textbooks seems to reflect the " $i j k$ is too formal" view, showing a complete absence of the $i j k$ representation (though using components through $\vec{A}_{x}, \mathbf{A}_{\mathbf{x}}$, or $A_{x}$ ), while frequently using the arrow representation in the introduction of vectors [10].

The objective of this work is to determine whether students in an algebra-based course, without any explicit instruction in the $i j k$ method, would perform better on vector addition and subtraction in the $i j k$ representation, similar to students in the calculus-based course reported in [9]. We also investigate whether this difference is uniform across a variety of relative vector orientations or restricted to a particular subset. In the next section we will describe the population studied, collection methods used, and the format of the vector questionnaire. We then present and analyze the results for oneand two-dimensional vector operation problems, and subsequently look at differences in performance for different relative orientations of vectors. Lastly, we end with conclusions and ideas for future study.

\section{METHODS}

Participants in this study were students enrolled in a first-semester (Mechanics) introductory physics course. The course was algebra-based (college algebra and trigonometry are pre-requisites), and is primarily populated by preprofessional life science students. There was a single lecture section consisting of 155 students, and the lecture comprised a mix of traditional and Peer Instruction methods, meeting 3 times per week with weekly online assignments [11]. The associated lab is not required for enrollment in the lecture. The author of this paper was the instructor for the lecture section under study.

The assessment was given as a required online assignment during the last week of class. Students were given full credit for completion, and were asked to explicitly consent to having 


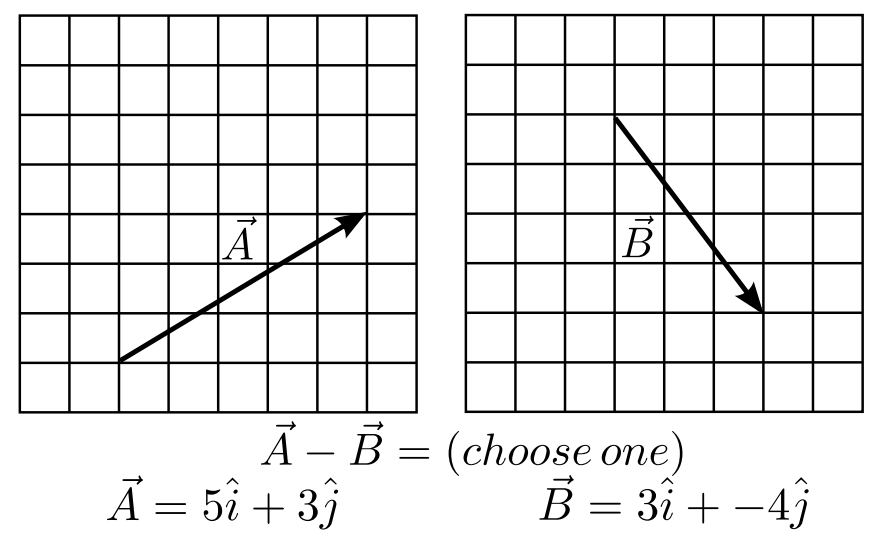

FIG. 1. Example of the arrow and $i j k$ formats used in the survey. Since only the vertical components have opposing signs, this was categorized as a "Yo" question.

their responses analyzed. All who did not answer "yes" (by answering "no" or leaving the response blank) were removed from the analysis. There were 120 participants in the study, giving a participation rate of $77 \%$. Students were randomly chosen to receive either the $i j k$ or arrow assessment, and the number of participants was $N=57$ for $i j k$ and $N=63$ for arrow. Students were not told if their answers were correct upon submission. They could complete the assignment at any location and any time during the week, and were given three hours to do so once they had viewed the research consent question.

The survey consisted of 28 multiple-choice questions, and each question contained a response box where students were asked to explain their answer. The questions contained a variety of one- and two-dimensional addition and subtraction tasks with varying vector orientations in the following order: six 1D addition questions, six 1D subtraction questions, eight 2D addition questions, and eight 2D subtraction questions. The vectors and tasks in each version of the survey ( $i j k$ or arrow) were identical and presented in identical order. A sample of the representations is given in Fig. 1. The vectors across operations were identical and presented in identical order (the third $2 \mathrm{D}$ addition question and the third $2 \mathrm{D}$ subtraction question used identical vectors). All questions were in a generic mathematical context, e.g., no deliberate physical context.

\section{RESULTS - OPERATION \& REPRESENTATION}

The results of the survey are shown in Fig. 2. Scores were higher in the $i j k$ format for addition and subtraction in both one and two dimensions, with only scores for onedimensional addition within one standard error. A 2-way ANOVA on the one-dimensional responses show that both the effects of vector operation and representation were significant. Scores were significantly higher in the $i j k$ format compared to the arrow format $[F(1)=6.9, p=0.0096]$, and significantly higher for addition compared to subtraction $[F(1,118)=60.8, p<0.0001]$. There was a significant interaction between the factors in one dimension $[F(1,118)=$

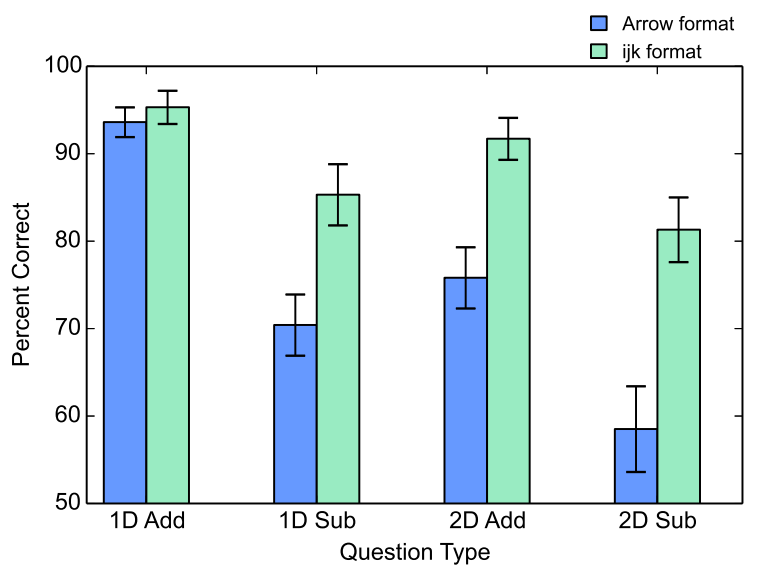

FIG. 2. Correct responses for one- and two-dimensional vector operations in the arrow and $i j k$ formats. Scores were higher in the $i j k$ format $(N=57)$ compared to the arrow format $(N=63)$, while scores for subtraction were lower than scores for addition. Error bars represent \pm 1 standard error (SE).

9.3, $p=0.003$ ], which can be seen in Fig. 2 with subtraction significantly more difficult than addition in the arrow format compared to the $i j k$ format. Scores for addition were 95.3\% ( $i j k$ ) compared to $93.6 \%$ (arrow), and for subtraction the scores were $85.4 \%$ ( $i j k$ ) compared to $70.4 \%$ (arrow).

In two dimensions, a 2-way ANOVA shows that both the effects of vector operation and representation were significant. Scores were significantly higher in the $i j k$ format compared to the arrow format $[F(1)=16.1, p=0.0001]$, and significantly higher for addition compared to subtraction $[F(1,118)=27.9, p<0.0001]$. In contrast to the onedimensional case, there was no significant interaction between vector operation and representation. Scores for addition were 91.7\% ( $i j k$ ) compared to $75.8 \%$ (arrow), and for subtraction the scores were $81.4 \%$ ( $i j k$ ) compared to $58.5 \%$ (arrow).

\section{A. 1D - Effect of Question Type on Correct Response}

The one-dimensional questions can be further broken down into subcategories that characterize the operation and vector orientation. Here we adopt the nomenclature from Ref.[9]: $p p p$ for a rightward vector added to a rightward vector ("positive plus positive"), $p p n$ for a rightward vector plus a leftward vector ("positive plus negative"), $p m p$ for a rightward vector minus a rightward vector ("positive minus positive"), and pmn for a rightward vector minus a leftward vector ("positive minus negative"). Students were given three questions in each category in the following order: $p p p, p p n, p m p$, and $p m n$. Student scores for both representations in each of these categories are shown in Fig. 3 along with a reproduction of the corresponding figure in Heckler et al. [9, Fig. 2].

Pairwise $t$-tests between categories within each representation show significant differences across vector operations regardless of orientation (subtraction is always harder than addition), but not across orientation within a given operation (sub- 

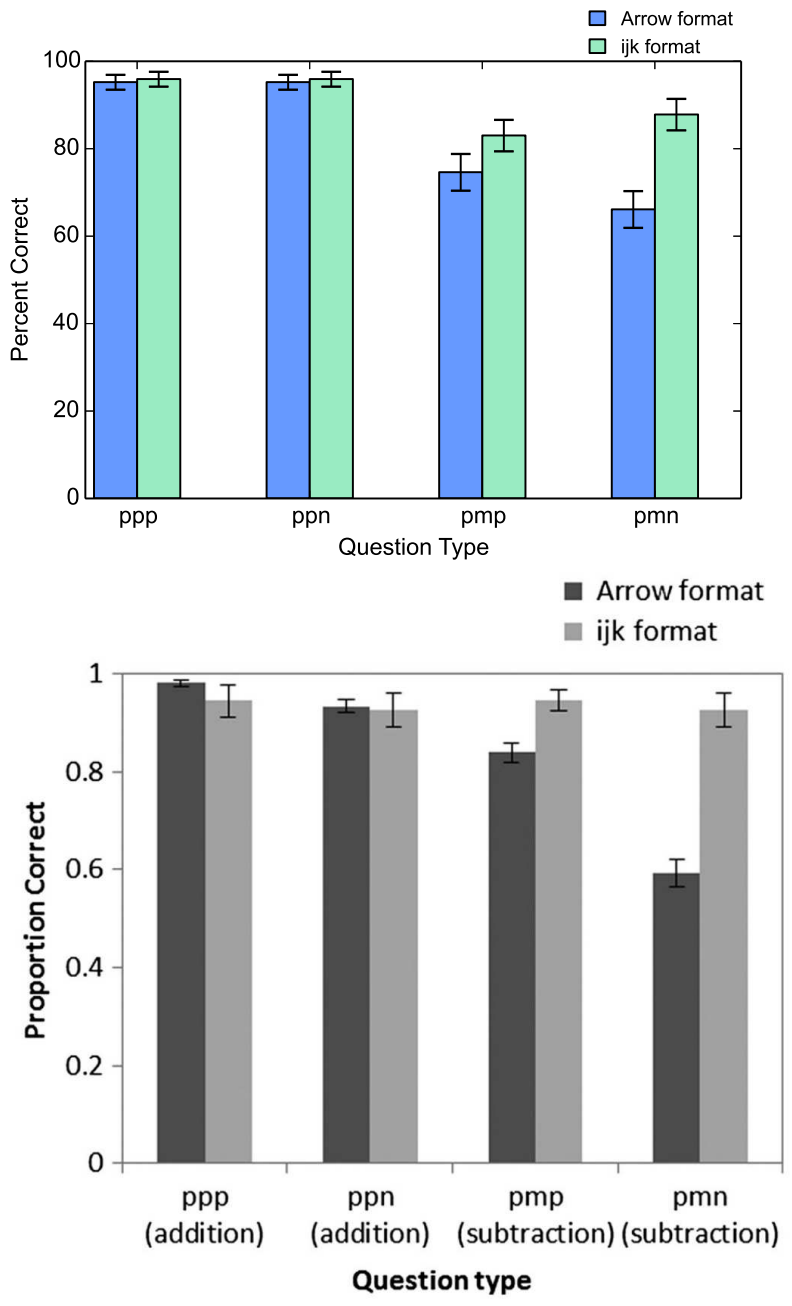

FIG. 3. Comparison of student scores by question type in one dimension. Top: student scores for the algebra-based course in this study. Bottom: a reproduction of Fig. 2 for student scores in a calculusbased course from Ref. [9]. Both populations show high scores for addition regardless of orientation or representation, while subtraction scores are lower in the arrow format, particularly for subtraction of oppositely-aligned vectors ( $p m n$ ). Error bars represent \pm 1 SE.

tracting two aligned vectors is not significantly different than subtracting two opposing vectors), with the $p$-values listed in Table I. Our findings parallel those of Heckler with $i j k$ scores higher than arrow scores regardless of category, but differ in that we find a significant difference between addition and subtraction in the $i j k$ format for the algebra-based students in this study [9]. Additionally, the only subtraction category that showed a significant difference between the arrow and $i j k$ format was pnm $[t(118)=3.8, p<0.001]$. These differences are likely due to the lower score on $i j k$ overall compared to Heckler's students, which may be due to the absence of $i j k$ instruction.
TABLE I. Left: $p$-values for pairwise paired $t$ tests across operation categories in the arrow representation. Right: $p$-values for pairwise paired $t$ tests across operation categories in the $i j k$ representation. The comparison between $p p p$ and $p p n$ could not be done in either representation due to identical means. ${ }^{*}$ indicate significance at the $p<0.05$ level after Bonferroni correction for multiple tests (five). $\dagger$ indicate significance at the $p=0.06$ level after correction.

\begin{tabular}{cccc|ccc}
\hline \hline & pmn & pmp & ppn & pmn & pmp & ppn \\
pmp & 0.08 & - & - & 0.18 & - & - \\
ppn & $<0.001^{*}$ & $<0.001^{*}$ & - & $0.01^{\dagger}$ & $<0.001^{*}$ & - \\
ppp & $<0.001^{*}$ & $<0.001^{*}$ & - & $0.01^{\dagger}$ & $<0.001^{*}$ & - \\
\hline \hline
\end{tabular}

\section{B. 2D - Effect of Question Type on Correct Response}

The two-dimensional questions can also be divided into subcategories. Here we categorize two vectors as "Aligned" (A) if both components have the same sign, "X Opposed" (Xo) if the horizontal components have opposite signs, "Y Opposed" (Yo) if the vertical components have opposite signs, and "Opposed" $(\mathrm{O})$ if both components have opposite signs. In all questions the first vector pointed "right and up". Students were given 2 questions per category in the following order; A, Xo, Yo, and O, first for addition and then for subtraction. Student scores in both representations for each orientation are shown in Fig. 4. Since the 2-way ANOVA for the two-dimensional questions showed no significant interaction between vector operation and representation, we analyze the performance across orientations separately for the $i j k$ and arrow representations.

Figure 4 shows that in the arrow representation there is no significant variation in student performance across vector orientation within a given operation (e.g., they perform the same on addition regardless of if the vectors are aligned). Performance on addition tasks was significantly higher than for subtraction for all orientations, using pairwise $t$-tests $(p<0.05)$ and correcting for multiple (four) comparisons.

In the $i j k$ representation, after Bonferroni correcting for multiple (six) comparisons, a significant difference in performance across orientation within operations was found between Xo (75\%) and $\mathrm{O}(89 \%)$ for subtraction [paired $t(56)=$ $3.24, p=0.002]$. This contrasts with the arrow representation where there were no significant differences across orientations within a given operation. An additional (and curious) difference is that there is not a significant difference in student performance between addition and subtraction tasks within the "Yo" and "O" orientations (after Bonferroni correction). Figure 4 shows that performance on addition and subtraction tasks for $i j k-\mathrm{O}$ questions is nearly identical, which contrasts with the significant difference found in the analogous one-dimensional case between ijk ppn (96\%) and pmn $(88 \%)$ question types. These results suggest that performance of this student population is independent of the relative orientation of vectors for the arrow representation but not for the $i j k$ representation, and this comes after no explicit instruction on the $i j k$ method. 

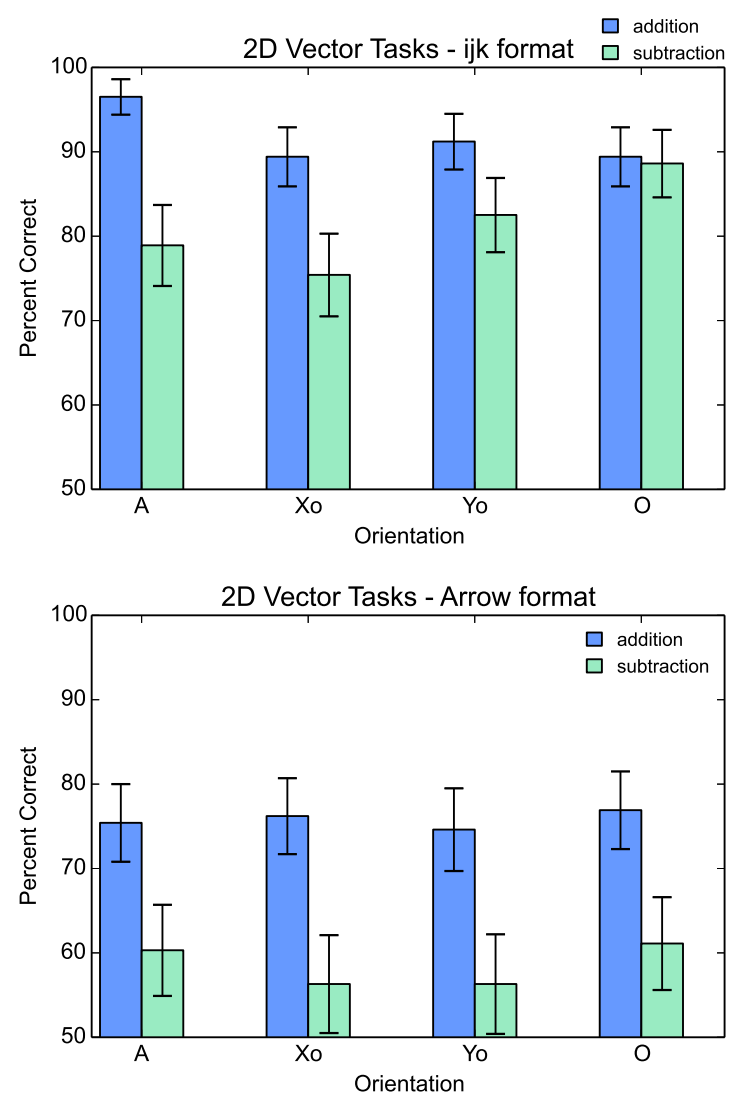

FIG. 4. Student performance on two-dimensional vector addition and subtraction questions in $i j k$ and arrow formats across different relative orientations of vectors. Error bars represent \pm 1 SE.

\section{CONCLUSIONS}

The main result of this study shows that students in an algebra-based introductory physics class perform better on vector addition and subtraction in one- and two-dimensional problems given in the $i j k$ format rather than the arrow format. This is perhaps unexpected given the lower mathematical preparation level of the students and the absence of any explicit $i j k$ instruction in the course. In light of this finding, the lack of $i j k$ or a similar method in many introductory textbooks at the algebra level is surprising [10].
We are careful to note, however, that we only tested the students on successful completion of the vector operations, not whether they could interpret their answers correctly in a mathematical or physics context, or if they could successfully translate between representations. It is possible that with the multiple-choice format of the exam the $i j k$ representation cued the students into an algebraic mode of problemsolving, "adding numbers" rather than thinking about vectors as a whole. Selection effects may also be present since not all students participated in the study. Even with this reserved interpretation, our results suggest a resource that could be utilized by instructors and students to assist with mastery of vector operations in both representations. This is supported by the evidence (for students in a calculus-based course) of a knowledge hierarchy between the $i j k$ and arrow representations [9].

For problems in one dimension, our results are in agreement with those that previously found no effect of vector orientation on student performance for addition (arrow and $i j k$ ) and subtraction $(i j k)$. While students did perform better on subtraction in the arrow representation of aligned $(p m p=75 \%)$ than opposing vectors $(p m n=66 \%)$, in agreement with $[8,9]$, our results did not rise to the level of statistical significance.

In two dimensions, we found that vector orientation in the arrow representation made no observable difference for addition or subtraction, but did make a significant difference in the $i j k$ representation for subtraction. We also found that student performance on vector addition and subtraction was not significantly different in the $i j k$ representation for vectors in which horizontal and vertical components had opposite signs. This counter-intuitive result, that students do not suffer a "subtraction penalty" for opposing $i j k$ vectors, but do in every other $i j k$ category, warrants further study.

Future work will investigate if the increased performance on $i j k$ remains when questions are posed in a physical context and the possibility of a knowledge hierarchy between the $i j k$ and arrow representations for students in an algebra-based introductory physics sequence.

\section{ACKNOWLEDGMENTS}

Thanks to Professors Mila Kryjevskaia and Warren Christensen for their guidance and questions in the preparation of this study and report. Thanks also to Dr. Jeffrey Boyer and Prof. Sara Anderson for helpful discussions on statistics.
[1] R.D. Knight, Phys. Teach. 33, 74 (1995).

[2] N.-L. Nguyen and D.E. Meltzer, Am. J. Phys. 71630 (2003).

[3] S. Flores, S.E. Kanim and C.H. Kautz, Am. J. Phys. 72460 (2004).

[4] P.S. Shaffer and L.C. McDermott, Am. J. Phys. 73, 921 (2005).

[5] J.M. Hawkins, J.R. Thompson, M.C. Whittman, E.C. Sayre and B.W. Frank, , AIP Conf. Proc. 1289, pp. 169-172.

[6] P. Barniol and G. Zavala, AIP Conf. Proc. 1289, pp 73-76.

[7] J.M. Hawkins, J.R. Thompson and M.C. Whittman, AIP Conf.
Proc. 1179, pp. 161-164.

[8] T. Wang and E.C. Sayre, AIP Conf. Proc, 1289, pp. 329-332.

[9] A.F. Heckler and T.M. Scaife, Phys. Rev. ST Phys. Educ. Res. 11, 010101 (2015).

[10] H.D. Young, P.W. Adams and R.J. Chastain, College Physics, 10th Ed. (Pearson, 2016); D.C. Giancoli, Physics: Principles with Applications 7th Ed., (Pearson, 2014); R. Knight, B. Jones and S. Field, College Physics 2nd Ed. (Pearson, 2010); N. Giordano College Physics 2nd Ed., (Brooks/Cole Cengage, 2013).

[11] E. Mazur, Peer Instruction (Addison-Wesley 1997). 